\title{
REFLEXIONES ACERCA DEL CARÁCTER DEL PENSAR EN SERENIDAD, DE MARTIN HEIDEGGER
}

\author{
Felipe Orellana ${ }^{1}$ \\ Centro de Investigaciones Históricas, Universidad San Sebastián
}

Recibido: 12.05.2018 - Aceptado: 06.07.2018

\begin{abstract}
RESUMEN
El pensar cobra gran importancia en la obra tardía de Martin Heidegger, momento en que sus meditaciones se plasman en un gran número de cartas, conferencias y ensayos dirigidos a la posibilidad de reflexionar sobre el ser, apartándose de la tradición filosófica de Occidente agotada en la metafísica. Es en esta época cuando aparece el discurso Serenidad, cuya relevancia radica en la actitud frente a la época técnica, cimentada en el pensar meditativo que se enfrenta al calculador. Será significativo, para estas consideraciones sobre el discurso mencionado, prestar especial atención a los recursos metafóricos y lingüísticos empleados por Heidegger, de modo tal que el concepto de autoctonía toma gran peso. Dicho concepto revela la posibilidad de pensar meditativamente en nuestros días y viene a enriquecer la reflexión acerca del carácter de este pensar en el discurso de Heidegger.
\end{abstract}

Palabras clave: Martin Heidegger, pensar, Serenidad, pensar meditativo, autoctonía.

\begin{abstract}
Thinking takes a great relevance in the late work of Martin Heidegger, in which his meditations covered a great number of letters, conferences and essays dedicated to the possibility of reflecting on being departing from the Western philosophical tradition. In this period appears Releasement, whose relevance lies in the attitude towards the technical era, based on the meditative thinking as opposed to the calculative one. It will be significant, in order to develop these considerations about this work, to pay special heed to the metaphoric and linguistic resources used by Heidegger, especially to the concept of autochthony, which reveals the possibility of meditative thinking nowadays and comes to enrich the reflection about the character of this thinking within Heidegger's work.
\end{abstract}

Keywords: Martin Heidegger, thinking, Serenity, meditative thinking, autochthony.

\footnotetext{
1 felipe.i.orellana@gmail.com
} 


\section{INTRODUCCIÓN}

El discurso Serenidad (Gelassenheit) ${ }^{2}$ pronunciado en 1955, pertenece a las obras y pensamientos tardíos de Martin Heidegger, destacados por apartarse del lenguaje científico de Occidente, para centrarse en uno que recurre a la metáfora y la poesía, llegando incluso a una variante mística ligada al silencio (Rivera 1998; Muñoz 2006; Garrido 2015). En esta época, el filósofo alemán pretendió el acercamiento al ser, ya no solo desde el Dasein como puerta de entrada, sino que, recurriendo al develamiento de lo oculto en el ser, buscando su claro (Lichtung) mediante el pensar (Garrido 2014). Entonces, los escritos, poemas, cartas y el discurso que analizamos en este trabajo, se centraron en la reflexión y meditación sobre el pensar mismo y su relación con el ser. Queda claro este propósito en obras como Desde la experiencia del pensar (1947) durante su estadía en la cabaña de Todtnauberg; Holzwege (1950), que según los comentarios de Leyte (1996) ha sido incluso más leído que Ser y tiempo, y también en ¿Qué significa pensar?, cursos de invierno y verano que dictó durante 1951 y 1952, donde se palpa la calidad libre, pero aguda y profunda de su pensamiento.

Así también lo hizo Heidegger en Serenidad, donde tomó distancia de los círculos académicos y pretendió contribuir hábil y magistralmente al pensar llevando sus reflexiones a un pequeño y tradicional pueblo en el

2 Para el análisis de este discurso, utilizamos dos versiones de su traducción al castellano. La principal, es la publicada en Revista Colombiana de Psicología núm. 3 (1994), que a su vez es tomada desde ECO: revista de la Cultura de Occidente núm. 4 (1960), donde la traslación estuvo a cargo de Antonio de Zubiaurre. En segundo lugar, revisamos la versión de Yves Zimmermann, publicada por Ediciones del Serbal, Barcelona (1994) para el necesario contraste, de lo cual, debemos hacer notar que hay dos motivos principales por los que utilizamos la realizada por de Zubiaurre en desmedro de la segunda. El primero, es que este último traduce la palabra bodenständig por autoctonía y no por arraigo - como hace Zimmermann-. Autoctonía es relevante en este artículo, y es empleada para el desarrollo de su propósito, pues el autóctono es quien nació en el lugar que reside, que partió su vida donde aún la continúa. El arraigo, por otra parte, es el afecto de quien por voluntad, gusto o interés se siente ligado a un lugar, que no necesariamente implica haber estado desde siempre allí. Desde ya anunciamos, que el carácter del pensar en Serenidad, se fundamenta en la autoctonía nuestra en él; fuera del interés o el gusto por pensar, hemos nacido y permanecemos aún en el pensar. Además, cabe considerar que la versión traducida al inglés realizada por Anderson y Freund (1966), rescata el concepto de autoctonía (autochthony). En segundo lugar, Zimmermann traduce la palabra Brachland por yerma, que es más bien un terreno estéril, en desmedro de la palabra que prefiere de Zubiaurre, pues la traduce como barbecho, lo que es también de suma importancia para este trabajo, pues dicho concepto implica riqueza y fertilidad a la vez que reposo. El tratamiento de esto se encuentra primeramente anunciado por la nota número 3 y luego se desarrolla con mayor detalle en la sección cuatro (4) de estas reflexiones. Por lo tanto, las citas expuestas en este artículo son tomadas de la versión que ofrece la Revista Colombiana de Psicología 3 (1994). 
suroeste alemán. Se trata de un breve y hondo llamado al pensar, advertencia de la época técnica a los vecinos de Meßkirch.

Entonces, nos proponemos identificar el carácter del pensar presente en el discurso mencionado. ¿Cuál es el sentido que tiene el pensar? ¿Quiénes y cómo pueden pensar? Son las preguntas que orientan nuestras reflexiones al respecto de este breve discurso. La razón de esto, es que consideramos que el pensar es el objeto de mayor importancia aquí, como ya venimos anunciando, pues gran parte de las obras que el autor realizó a partir de 1930 se dedicaron a esto; el pensar como medio de acceso al ser. Siguiendo con esto, no es casualidad que, el título de la primera traducción de Gelassenheit al inglés haya sido Discuourse on Thinking (Discurso sobre el pensar), traducido por John M. Anderson y Hans Freund, publicado en Harper and Row Publishers (New York) el año 1966. Esta visión es compartida también por Cordua (1999), cuando se enfoca en ello, incluso más allá de la misma serenidad.

Hará falta entonces, un examen profundo a raíz del discurso mencionado, contextualizando y sirviéndonos del estilo metafórico, además de las herramientas lingüísticas empleadas de nuestro filósofo, por ello la preferencia de una versión por sobre otra. Para esto, echaremos mano a las bases metodológicas y conceptuales acerca de la fenomenología en trabajos anteriores como Ontología (Hermenéutica de la facticidad) (1923), Ser y tiempo (1927) y Carta sobre el humanismo (1946), además de la utilización de nociones que en la obra de 1927 aparecen, como lo son el uno (das Man), ser-ahí (Dasein), autenticidad (Eigentlichkeit) y publicidad (Öffentlichkeit). A pesar de que tanto el tratamiento de estos conceptos y de las bases del método fenomenológico son expuestas esquemáticamente, nos dan un sustento especial para entender el pensamiento del filósofo hacia la segunda parte de su carrera intelectual.

Finalmente, cabe hacer hincapié en cuanto a las interpretaciones y problemáticas que suceden sobre el mencionado discurso. Antes que todo, destacamos que la traducción desde el alemán (Gelassenheit) por Serenidad, ya reviste una gran complejidad por no lograr rescatar todo el sentido que tiene en la lengua original. Esto ha provocado discusión, llevando a algunos estudiosos a re- interpretar el concepto. Tal es el caso de lo que realiza Cordua (1999), que como mencionamos, prefiere el término desasimiento, recatando el carácter teológico original presente en él. Además de lo que sucede con la traslación al castellano, las dificultades persisten para las interpretaciones del concepto en inglés, traducido como releasement, lettingbe o detachment (Anderson - Freund 1966; Shürmann 1973; Parente 2006). No obstante, consideramos que la utilización de Serenidad, canónica por lo demás, no perjudica el objetivo de nuestra propuesta. 
A pesar de los problemas en cuanto a las traducciones, queremos hacer mención de la interpretación elaborada por Cordua (1999) cuando propone que el concepto serenidad debe ser vertido por desasimiento, que incluso engloba a la misma serenidad. De esta forma, la filósofa chilena comenta: "Desasido es el que se abstiene de prender a lo que es y evita dejarse prender por ello. Pero desasimiento es la acogida serena, desaprensiva, de lo que libremente se muestra" (Cordua 1999, 35).

Lo anterior, podemos contrastarlo con lo que argumenta Gutiérrez (2003), cuando menciona que la serenidad no es una actitud ante las cosas o al mundo, sino que es una pre- disposición, una forma de dejar al ente ser; solo de esta forma se deja que las cosas aparezcan. Cordua, establece que la relación con el ser es de tal grado difícil, que solo el pensar puede acércanos a él, por lo que, desasirse de las cosas, dejando que el ser sea, es a la vez pensar. Mientras que Gutiérrez considera que no se trata de una actitud, Cordua sí lo hace, pero ambos concuerdan en que la serenidad o desasimiento produce que el ser sea. La diferencia entre estos dos autores, es que la filósofa chilena reflexiona con ahínco sobre el pensar en el discurso aludido, considerando que no se encuentra siempre presente en nosotros, y que solo es logrado en la medida que nos desasimos de las cosas para acercarnos al ser.

\section{SERENIDAD: DISCURSO Y PROBLEMA}

Meßkirch, una pequeña ciudad al suroeste de Alemania, ha brillado para los alemanes, pues es la patria de Conradin Kreutzer, destacado pianista y compositor romántico, a la vez que de Martin Heidegger, filósofo.

En 1955, se cumplían 175 años desde el nacimiento del músico y el pueblo suabo se reunía para conmemorarlo en lo que se denomina una fiesta conmemorativa (Gedenknfeier). Por supuesto, lo conmemorativo y sus fiestas en nuestro hemisferio y cultura también se dan, pues allí recordamos héroes militares, hechos históricos y también el nacimiento de hijos ilustres. La cultura (Kultur) alemana, da espacio para la patria y el sentimiento de pertenencia regional, pues hubo tantos reinos como pasado común o singular, que formaron el carácter variopinto de dicho pueblo ancestral. En este contexto de riqueza cultural, se reunieron los nacidos en Meßkirch, con música y ánimo festivo para el recuerdo (Andenken) de su músico destacado. Heidegger fue el elegido para el discurso conmemorativo (Gedenkrede), herramienta vital para acercar el pensar y la filosofía a los presentes.

Como anunciamos, la conmemoración de los destacados e ilustres, aquí o allá, es sinónimo de enaltecer la cultura, la historia común y sus riquezas. El hecho está, en que aquí se hace patente el supuesto hecho de que el pueblo se reúne para pensar, recordando lo que los antecede, lo que los une y de lo 
que los enorgullece. Sin embargo, Heidegger apunta a estos sentimientos, intentando encontrar lo auténtico de esta inautenticidad pública que dice pensar sin tener conciencia de ello. Heidegger parte, luego del protocolar agradecimiento, desde el análisis de la palabra fiesta conmemorativa (Gedenknfeier), cuya raíz en alemán está en pensar (denken). El filósofo pregunta y afirma: "La fiesta en la que se toca y canta ¿es ya una fiesta conmemorativa (Gedenknfeier), una fiesta en la que pensamos (denken)? Presumimos que apenas lo sea" (Heidegger 1994, 23).

¿Era preciso que, en este momento de alegría y de fiesta, el pensamiento se haya hecho tan profundo e incluso doloroso? Veremos.

Heidegger, no escatima en ponerse a sí mismo como el que lleva a los demás a pensar, queda claro cuando dice: "Por ello los organizadores del acto han puesto en el programa un discurso conmemorativo (Gedenkrede). Y ese discurso debe ayudarnos a pensar realmente en el artista festejado y en su obra" (Heidegger 1994, 23). Y lo cierto, es que a nuestro parecer así sucede, lentamente lleva a los reunidos a poner en duda su propósito, incluso su propio lenguaje, a filosofar de una manera especial revestida de una gran simpleza.

En efecto, el maestro menciona que "no nos engañemos (...) todos nosotros somos a menudo pobres en pensamiento; todos nosotros, con harta facilidad, estamos faltos de pensamientos" (Ibídem). Su inclusión en el problema es parte del propósito de hacer pensar, y no simplemente hacer pensar en Kreutzer, sino que también hacer pensar a todos, incluido él.

Una vez establecido el problema - de que estamos faltos de pensamiento- Heidegger lo aborda y otorga las posibles vías de solución. El filósofo dice:

Pero, aun cuando estemos faltos de pensamientos, no renunciamos a nuestra capacidad de pensar. La necesitamos incluso indudablemente, aunque en verdad de una especial manera: haciendo que en la carencia de pensamientos quede en barbecho nuestra capacidad de pensar (Ibídem).

Permítasenos aclarar lo que es un barbecho, ${ }^{3}$ pues es época y lugar; terreno para el cultivo que no está siendo cultivado, sino que se deja descansar, donde la tierra halla su fuerza mineral apartándose del quehacer y dedicándose a su propia reconstrucción. A pesar de esta riqueza, el barbecho es lo olvidado, porque en la actividad de cultivar lo dejamos

3 Aquí está la primera justificación de la utilización de barbecho (Brachland) (traducción de Antonio de Zubiaurre) por sobre yerma (Zimmermann). El desarrollo de esto continúa en la sección cuatro (4). 
ignorado, y a pesar de esto, es más rico que cualquier otro suelo, pues por eso queda allí guardado y esperando. El filósofo continúa: "Ahora bien, en barbecho solo puede estar lo que en sí constituye una base para el crecimiento" (Ibíd.). En efecto, siguiendo con la ejemplificación, en una autopista nada puede crecer y jamás podrá ser barbecho, pero, aun le damos utilidad, nos sirve para algo. ¿Debemos usar el barbecho? ¿Heidegger nos pide que no nos enfoquemos en la utilidad y demos espacio a la riqueza de nuestro suelo? ¿A qué apuntan estas alegorías de suelo y tierra? Veremos.

Todo esto es preparatorio para establecer la diferencia entre el pensamiento calculador (das rechnende Denken) y la meditación reflexiva (das besinnliche Nachdenken), que son la base para las vías de interpretación que se han dedicado al análisis de la época técnica y los tipos de pensar frente a ella. ${ }^{4}$ Vía filosófica importante, que el mismo Heidegger abordó en otros escritos y conferencias. ${ }^{5}$ Pero lo que a nosotros nos concierne ahora, es abordar el trasfondo de este discurso y los propósitos que conllevan estas reflexiones más allá de lo que es patente en él. Estamos ante la reflexión del pensar mismo, y la forma de ser abordado será realizando una lectura fenomenológica, previa explicación esquemática de dicho método.

\section{APROXIMACIONES AL MÉTODO FENOMENOLÓGICO DE HEIDEGGER A PROPÓSITO DEL DISCURSO SERENIDAD}

Para revelar el carácter que tiene el propósito de lo que Heidegger hizo en este discurso, precisamos una visita a las bases metodológicas de su pensar: la fenomenología. Dentro de esto, mencionaremos rápidamente que se trata de la herencia de una escuela importante, fundada por el maestro de nuestro filósofo, Edmund Husserl, en Investigaciones Lógicas I y II (1900-1901), cuya continuación explicativa se haya en Ideas relativas a una fenomenología pura y una filosofía fenomenológica (1913) y en La crisis de las ciencias europeas y la Fenomenología Trascendental (1936). Husserl expresó en el prólogo de la obra de 1913, que su método no fue entendido, y se propuso a reelaborar su fenomenología poniendo énfasis en la cuestión del método y ahondando en lo que es ir a las cosas mismas. Pero, hacemos hincapié en la distancia que separó a Heidegger con Husserl, pues el primero dijo: "Mas las Investigaciones Lógicas no se entendieron; acaso ni hoy en día se entiendan” (Heidegger 2000, 95). Ahondaremos en este alejamiento con el maestro.

Husserl, con su fenomenología, intentó contrariar la tradición filosófica, acudiendo al rigor y abstracción de la lógica matemática, dedicándose a la esfera del interés lógico, porque según él, su método devino

${ }^{4}$ Vid.: Acevedo, Jorge. 2016. Heidegger y la época técnica. Santiago: Universitaria; Acevedo, Jorge. 2014. Heidegger: existir en la era técnica. Santiago: Ediciones UDP.

${ }^{5}$ Vid.: Acevedo, Jorge (Ed.). 2017. Filosofía, Ciencia y Técnica. Santiago: Universitaria; Heidegger, Martin. 2010. Caminos de Bosque. Madrid: Alianza Editorial. 
del estudio de la geometría principalmente; de ahí, se propuso "producir constructiva-unívocamente todas las formas ideales en absoluto concebibles en un método a priori, sistemático, omniabarcador" (Husserl 2008, 69).

La idea de la fenomenología para Husserl fue el establecimiento de un método para una filosofía sistemática y científica. Sin embargo, Heidegger redireccionó la fenomenología hacia una de carácter hermenéutico, no realizando otra fenomenología, sino que "pensar la esencia de la fenomenología de manera más originaria, para de este modo volver a integrarla propiamente en su pertenencia a la filosofía occidental" (Heidegger 2000, 95). La fenomenología para él debía ser reintegrada en el pensar, y solo así sería más originaria. Por lo tanto, esta debía ser un modo de investigar: "hablar de algo tal como ese algo se muestra y solo en la medida en que se muestra" (Ibídem). Filosofía fenomenológica es, por consiguiente, un error, pues convertir el pensar en un modelo - en específico como el que planteó Husserl— es anti-fenomenológico.

La fenomenología es un cómo: "actualiza los objetos en la intuición y solo habla de ellos en la medida en que están ahí en tal intuición" (Heidegger 2000, 96). Estas aseveraciones las elabora a partir de una dura crítica a su maestro y a las corrientes intelectuales del momento que malinterpretaban la fenomenología. Heidegger realiza una reivindicación de ella, añadiéndole, como mencionamos, el carácter de hermenéutica. ${ }^{6}$ Este fue el rasgo que terminó por fundamentar la diferencia entre Husserl y Heidegger: que el radicalismo pretendido por Husserl no fue tal, pues la reducción del problema a la existencia, y con ello al tiempo y la historicidad (como hace Heidegger) ahondaron radicalmente en el problema y contradijeron totalmente a lo trascendental buscado por Husserl. De aquí que Heidegger propuso la supremacía del Dasein para acceder al ser, pues es el ente que en su ser le va su propio ser; su ser no le es ajeno. Por tanto, une la ontología y la fenomenología en tanto que hermenéutica.

Por último, el trecho se hizo manifiesto cuando aclaró: "Husserl aportó al respecto de cosas decisivas. No obstante, hay que saber oír y ser capaz de aprender. En vez de eso lo que se encuentra es funcionamiento rutinario en el desconocimiento de las cosas" (Heidegger 2000, 101).

\section{a. Algunos fundamentos de la fenomenología heideggeriana}

Es en su magnum opus (Ser y tiempo), donde está la base -aunque incompleta- del método fenomenológico. ${ }^{7}$ Todo parte en la distinción y

6 Vid.: Heidegger, Martin. 2000. Observación previa, fenómeno y fenomenología. En: Ontología (Hermenéutica de la facticidad, 91-101. Madrid: Alianza Editorial.

${ }^{7}$ La idea de lo incompleta que resultó ser la aproximación conceptual acerca de la diferencia ontológica en Ser y tiempo, es tratada en: Pulido, Jorge. 2015. "<<La ontología solo 
conexión entre el ser y el ente, es decir, la diferencia ontológica (ontologische Differenz), donde tiene también su raigambre la fenomenología (Pulido, 2015).

Por supuesto, y siguiendo el análisis de Pulido (2013; 2015), la raíz de fenomenología nos direcciona a la palabra fenómenos (del griego paıvó $\mu \varepsilon v o v$ ), que "es la totalidad de lo que yace a la luz del día o que puede ser sacado a la luz" (Heidegger 2002, 52). Sin embargo, está aquí presente la posibilidad de que el fenómeno se muestre como lo que no es en sí mismo; esto es la doble significación de fenómeno: lo que se muestra en sí mismo y lo que se muestra como lo que no es (apariencia, Schein). Este doble significado implica a su vez "el ocultarse, sin dejar nada atrás, sin suponer algo que no pueda llegar a mostrarse" (Pulido 2015, 101). Es la mostración que se da, pero ocultándose; se reduce a su ocultación.

Este movimiento del fenómeno, que aparece y se oculta, es la dinámica óntico-ontológica, y el ser (Sein), es de hecho esa dinámica, por lo que apariencia (Schein) es el concepto fenomenológico para fenómeno (Pulido, 2015). De aquí, se desprende que fenomenología entendida desde Heidegger, es el método y capacidad de ver esta dinámica óntico-ontológica, a la vez que mostrando al ocultar y ocultando al mostrar. Es esto lo que le dio gran originalidad a la labor filosófica del pensador alemán, a la vez que gran dificultad, donde el ser constituyó el tema central de su filosofía, teniendo este un carácter metahistórico que a su vez procede auto-ocultándose en su acontecer (Bech 2001; Garrido 2015).

Este carácter del ser, que va ocultándose a través del tiempo, llevó a hacer pensar a Heidegger que la historia de la filosofía ha sido justamente eso, la persecución, nunca lograda, del entendimiento del ser y su sentido (Bech 2001). No obstante, el pensador alemán dio una alternativa para descubrirlo: "el pensar es al mismo tiempo pensar del ser, en la medida en que, al pertenecer al ser, está a la escucha del ser" (...) "que el pensar es, significa que el ser se ha adueñado destinalmente de su esencia" (Ibidem, 16). Es esta la relevancia que tiene el pensar en Heidegger, medio de acercamiento al ser que es tratado con profundidad en la citada Carta sobre el humanismo, publicada en 1947 y dirigida a Jean Beaufret.

Por lo pronto, nos queda entender cuál es el carácter del pensar en Serenidad, donde se relacionan el ser y el pensar en esta dinámica que venimos enunciando.

es posible como fenomenología>>. En torno a la fenomenología de Martin Heidegger". Franciscanum 163: 87-123. 


\section{b. Fenomenología en Serenidad}

La aclaración de lo que Heidegger entiende por fenomenología es vital, pues Serenidad fue elaborado bajo el rigor del método fenomenológico. El pensar es aquí un fenómeno ( $(\alpha \imath v o ́ \mu \varepsilon v o v)$ inmerso en la dinámica óntico-ontológica de la apariencia (Schein): lo que se muestra en sí mismo y lo que se muestra como lo que no es.

Entender el pensamiento heideggeriano desde el rigor fenomenológico es tarea dura, pero vital para comprender lo que en su simple discurso estuvo oculto, en barbecho.

Al parecer, queda iluminado por el mismo Heidegger que carecemos de pensamientos porque yace en barbecho la capacidad de pensar y al parecer también, el discurso se orienta a animarnos a pensar y a mantenernos en serenidad y abiertos al misterio en medio de esta época técnica. ${ }^{8}$ Pero ¿qué está oculto en el discurso?

Cuando Heidegger aborda explícitamente lo que es serenidad, es para referirse a la era técnica, donde el dominio es de la ciencia atómica, la cual no piensa su propia época. Donde lo técnico embarga al hombre de su tierra, que es lo más propio y cercano a él: el pensar. Serenidad (Gelassenheit) es una actitud de conciliación, una sabia habilidad de dejar penetrar lo técnico en nuestras vidas, pero a la vez evitar que nos afecten. En palabras de Heidegger: "Podemos dar el sí a la ineludible utilización de los objetos técnicos, y podemos a la vez decir no en cuanto les prohibimos que exclusivamente nos planteen exigencias, nos deformen, nos confundan y por último nos devasten" (Heidegger 1994, 27).

Este sí y no ante la época y medio en que vivimos, no solo guarda aquí una advertencia sobre la radio, la televisión, los celulares inteligentes o el internet. Lo que Heidegger expone aquí, es una deformación que podría volverse radical, pues podría afectar lo que somos: pensadores autóctonos. Además, el sí y el no son a la vez un guiño al carácter fenomenológico que envuelve la serena actitud, pues esta es al mismo tiempo el pensar mismo, ocurrido, como ya quedó enunciado, por el embargo del ser que se deja mostrar; es a su vez el sí y el no del ser mismo, que se oculta y se muestra, al igual que el pensar.

8 La apertura al misterio es definida como "la capacidad en virtud de la cual nos mantenemos abiertos al sentido oculto en el mundo técnico" (Heidegger 1994, 27). Debe ir junto con la serenidad y es el estar atento, con mirada fenomenológica, a lo oculto de la época técnica, pues ella como fenómeno aparece: se muestra ocultándose. 


\section{LA AUTOCTONÍA DEL PENSAR: LO QUE ES PROPIO DE NOSOTROS}

No es casualidad la utilización del término autoctonía (bondeständig) para poder explicar el pensar. Heidegger recurre a esto, pues la fiesta conmemorativa de la patria de Meßkirch, es de hecho, la patria de él y de los ahí reunidos. Entonces, a propósito de que lo técnico invade nuestras vidas y nos despoja de lo que es más propio en nosotros (nos exilia de nuestra patria), Heidegger recurre a las palabras de Johann Peter Hebel: "Somos plantas, queramos o no confesarlo de buena gana, que debemos salir de la tierra para florecer en el éter y poder dar frutos" (Heidegger 1994, 24). El filósofo, continúa y amplía las palabras de Hebel: "Donde ha de crecer una obra humana verdaderamente gustosa y bienhechora, el hombre tiene que alzarse desde la profundidad del suelo patrio hasta el éter" (Ibídem).

Esta reflexión a partir de las palabras poéticas de Johann Peter Hebel, no es solo acerca del deber de mostrar lo mejor en cuanto que obras, enraizados en nuestra patria, como lo hiciera Kreutzer o Heidegger. Una vez más, el filósofo fue lejos de "conmemorar" y fue a conmemorar realmente. No solo estuvo hablando de la obra de un hombre o del deber, sino que hay oculto, además de lo iluminado, una reflexión sobre el pensar mismo.

En efecto, suelo (boden) es la raíz de autoctonía (bodenständig). Hay un suelo nuestro, que no es solo la patria en el sentido de lugar de nuestros padres y de nacimiento, pues esto aún es externo y lejano. Heidegger dice: "Más aún, la pérdida de la autoctonía no está causada solo por circunstancias y destinos externos, ni se debe únicamente al abandono y al modo superficial de la vida de los hombres" (Ibíd.).

Aquí la reflexión comienza a centrarse en la época técnica, guardando su sentido fenomenológico de luz-oscuridad. Mas, lo que nos interesa, es el carácter de esta patria y suelo propio, más allá de lo externo. Heidegger deja entrever que hay una capacidad inherente en todos nosotros, tan cercana y propia que es la tierra misma donde echamos las raíces y salimos al éter como dijera Hebel, que según Heidegger es: "el aire libre del alto cielo la abierta región del espíritu" (Ibíd.).

¿Qué es lo más propio del hombre? ¿Cuál es la patria del hombre? El pensar, sin duda. Pero ¿debemos ser académicos al pensar? ¿Ser maestros en filosofía, versados en la tradición filosófica? Al parecer no. Entonces cabe otra pregunta, ¿debemos ser o hacer como Heidegger? Y esto es más difícil de responder, pues el mismo Heidegger fue maestro de filosofía, conocedor profundo de la tradición filosófica, tanto así que se dio el lujo de ir contra ella, incluso contra su propio maestro. Sin embargo, no es casualidad que él se haya incluido en la sentencia de que estamos faltos de pensamiento: "Todos nosotros, incluidos aquellos que pensamos ya por pura exigencia profesional, todos nosotros somos a menudo pobres en pensamiento" 
(Heidegger 1994, 23). El pensar no está en la academia o en los libros de filosofía. El pensar tampoco está en los filósofos y, sin embargo, yace ahí de igual forma. Continuemos:

Al igual que podemos quedarnos sordos solo porque oímos, al igual que llegamos a viejos solo porque fuimos jóvenes, lo mismo podemos volvernos pobres en pensamiento, o hasta faltos de pensamiento, por razón de que el hombre, en el fondo de su ser, posee la capacidad de pensar (...) y porque está destinado a pensar (Heidegger 1994, 23).

Más allá del tono poético con que Heidegger aborda la cuestión, se puede aquí evidenciar la dinámica óntico-ontológica en la que cae este pensar como apariencia y fenómeno. La labor fenomenológica es seguida aquí con tenacidad, Heidegger piensa sobre lo iluminado y lo oculto del pensar mismo. Estamos en fuga del pensar, no solo por la época técnica que nos embarga de ello, sino que, porque yace en el fondo la capacidad misma de pensar, que es nuestro suelo patrio, más propio y próximo, esto es "porque el hombre es el ser pensante, o sea reflexivo" (Ibídem, 24).

Entonces, somos habitantes autóctonos del pensar, lo que lleva consigo también el carácter destinal de éste: "el ser se ha adueñado destinalmente de su esencia" (Heidegger 2000, 16). Este pensar meditativo, que es principio y destino, no puede ser arrancado, por más expuestos que quedemos a lo técnico, porque incluso el pensar calculador es a su vez pensar. La arenga del maestro es darnos cuenta de que yace, muchas veces oculta, la capacidad de pensar, que no es solamente un asunto práctico, de gusto o de profesión, pues aquellos también pueden llegar a no pensar. Hemos nacido y vivimos aún en el pensar, que es más que el sentimiento de arraigo, el gusto, o la dedicación profesional por él, sino que es tierra nuestra, somos habitantes autóctonos del pensar.

Pareciera ser que lo de Heidegger es una demanda, algo así como un imperativo ético de llevarnos a una vida de reflexión y meditación, mas, a nuestro parecer, y como en el resto de su obra, no está presente un sentido ético estrictamente hablando. El pensar, para referirnos solo a Serenidad es la patria nuestra, lo más próximo. No solo se trata aquí de algo que hay que ejercer, sino que solo es necesaria la apertura a ello, que es apertura a nosotros mismos. Esto guarda una simpleza inimaginada, que solo por el rigor del pensar heideggeriano tenemos acceso.

En vista de esto, hace falta retomar una interrogante anterior, ¿es necesario ser maestro en filosofía para pensar? La respuesta definitivamente es que no, pues aún los maestros pueden estar lejos de él. Siguiendo con 
nuestro escudriñamiento fenomenológico sobre el discurso, Heidegger dice: "la mera meditación se encuentra flotando sobre la realidad cuando menos lo espera" (Heidegger 1994, 24). Esto de mera meditación flotante, es lo que se dice sobre la reflexión y la meditación, es la fuga misma del pensar. Para esto, es importante el empleo de se, pues estamos ante uno de los caracteres más propios del Dasein: el refugio en el uno (das Man).

Corremos del pensar porque somos naturalmente inauténticos. Decimos lo que se dice en torno a la meditación y al pensar, porque esto "no sirve para salir adelante en los negocios. No aporta en nada a la práctica cotidiana (Durchführung der Praxis)" (Ibídem). Lo que se dice es el uno, lugar donde el Dasein se oculta: das Man. Heidegger continúa su análisis de lo inauténticos que somos al fugarnos del pensar: "Y se dice, en fin, que la mera meditación, la constante reflexión, es demasiado "elevada" para el entendimiento normal" (Ibídem). En la publicidad (Öffentlickheit), solemos apartarnos de la reflexión meditativa, o sea del pensar, porque se lo atribuimos a la academia o a los filósofos de profesión. Se entiende que este mero pensar flota en el aire, que el que piensa y medita no tiene los pies en la tierra (en el suelo). Lo que se olvida, es que nuestro suelo autóctono es justamente el pensar, solo que dejado en barbecho.

Se entiende que negar el ocio y dedicarnos al negocio es lo productivo, así nos dedicamos la mayoría del tiempo al cultivo del suelo que creemos nos dará beneficios. Y de hecho así es, ¿pero dejaremos que esto nos deforme y tenga total incidencia en nuestras vidas? Claramente, este es el llamado a la actitud de Serenidad. ¿Qué está en barbecho? Pues, el pensar, esperando y en desuso, pero aún así rico y fértil.

Entonces, ¿para quién es el pensar? No es para los que se dedican a ello de forma profesional, ni reside en los libros ni en la academia. ${ }^{9}$ El verdadero pensar se viste de una simpleza tal, que lo dejamos olvidado en barbecho. Es la dinámica óntico-ontológica de este, lo que provoca que lo dejemos ignorado y oculto. Porque aun cuando pensamos calculantemente, pensamos de todas formas (lo iluminado), queda entonces lo oscurecido de este pensar, que es sin duda difícil y requiere mayor esfuerzo. Heidegger dice: "Cualquiera puede seguir a su manera y dentro de sus límites los caminos de la meditación (...) Basta con que nos demoremos en lo próximo y reflexionemos en lo más próximo, en lo que a nosotros, a cada cual, aquí y ahora nos atañe" (Heidegger 1994, 24).

La invitación es a pensar, pero no como se hace, sino auténticamente. El pensar auténtico es el pensar meditativo, que transcurre incluso en la

${ }^{9}$ Esta idea es la continuación de lo que Heidegger hablaba a sus estudiantes en ¿Qué significa pensar? (Heidegger 2005). Allí, exponiendo sus ideas acerca del dominio técnico, elaboró una crítica a los centros de investigación y la academia en general, los cuales se ha adjudicado el hecho de pensar, cuando en realidad estos no lo hacen. 
cotidianidad, en nuestro propio suelo, nuestra propia patria "en el presente instante universal" (Ibídem). La reflexión meditativa es para todos por nuestra naturaleza, origen y destino, y por la apariencia del pensar como fenómeno; lo oculto y aparente del pensar es la fuerza misma que nos puede llevar al pensar. El que esté oculto, el dejarlo oculto, es crucial para ponernos en vías del pensar.

\section{CONCLUSIONES}

A través del examen fenomenológico de la reflexión heideggeriana, podemos ver lo oculto tras lo iluminado del pensar del filosofo alemán, porque este a su vez pensó en términos fenomenológicos. El breve discurso analizado, sirve como muestra de que el ejercicio del pensar en Heidegger no solo fue orientado a los círculos académicos o a las publicaciones de tipo disciplinar, sino también a hombres y mujeres de un pequeño, tradicional y remoto pueblo suabo.

El pensar es auténtico cuando es meditativo, y cuando se desarrolla en y sobre lo más próximo que tenemos, que no es la tradición filosófica de Occidente agotada en la metafísica, sino en nuestro propio suelo, allí donde venimos echando raíces desde siempre y nos movemos cotidianamente. Así, este puede transcurrir en lo cotidiano y puede volverse una tarea de cada día como implica esta palabra (del lat. quoti-die), pues podemos conciliarlo con nuestro corriente quehacer, incluso si este está orientado a la técnica y a la labor, pues es el llamado de Serenidad.

Más allá de los recursos estilísticos que ofrece la fenomenología —en el discurso y el texto - envuelta toda ella en la llamativa dinámica que ofrece el fenómeno, es menester que no solo nos sintamos atraídos a ella para entenderla en la obra de Heidegger, pues vale incluso más apropiarnosla para nuestro quehacer filosófico, que no queda reservado para los maestros, sino que para todo aquel que quiera internarse en el pensar de sus cosas y asuntos próximos. El darnos cuenta del claro-oscuro del pensar, es también caminar en vías de él; nos volvemos filósofos cuando nos inquieta el captar que hay gran parte de nuestra capacidad de pensar allí olvidada, pero que yace en barbecho, nutriéndose y retrayéndose en su fuerza original.

El pensar no solo está presente en este discurso breve y llamativo, y proponemos la búsqueda fenomenológica del carácter de este a lo largo de la obra de Heidegger, pues es posible que su estilo de vida ligado a lo natural, al campo y a las costumbres, sea la consecuencia de ello. Consideramos que la labor del pensar, así como su tratamiento fenomenológico, nos lleva así a lo oculto de la obra del filósofo y por supuesto, de nuestra propia vida. 


\section{BIBLIOGRAFÍA}

Anderson, John - Freund, Hans. 1966. Discourse on thinking. New York: Harper \& Row Publishers.

Bech, Josep. 2001. De Husserl a Heidegger. La transformación del pensamiento fenomenológico. Barcelona: Edicions Universitat Barcelona.

Cordua, Carla. 1999. Filosofía a destiempo. Santiago: RIL editores.

Garrido, Juan. 2015. Lichtung: el claro del ser. Un estudio a raíz de las meditaciones del Holzwege. Agora: papeles de filosofía, 2: 161-177.

Gutiérrez, Antonio. 2003. "Arte y Gelassenheit: Estética, ética y lógica originarias en el pensar de Heidegger". Logos. Anales del Seminario de Metafísica 36: 153-186.

Heidegger, Martin. 1994. "Serenidad". Revista Colombiana de Psicología 3: 2228. Tomada de ECO: revista de la Cultura de Occidente 4 (1960). Trad. de Zubiaurre, Antonio. Disponible online: http://revistas.unal.edu.co/index.php/psicologia/article/view/15808

Heidegger, Martin. 1994. Serenidad. Barcelona: Ediciones del Serbal. Trad. de Zimmermann, Yves.

Heidegger, Martin. 1999. Ontología (Hermenéutica de la facticidad). Madrid: Alianza Editorial. Trad. de Aspiunza, Jaime.

Heidegger, Martin. 2002. Ser y tiempo. Santiago: Universitaria. Trad. de Rivera, Jorge.

Heidegger, Martin. 2000. Carta sobre el humanismo. Madrid: Alianza Editorial. Trad. Cortés, Helena y Leyte, Arturo.

Heidegger, Martin. 2005. ¿Qué significa pensar? Madrid: Trotta. Trad. de Gabás, Raúl.

Husserl, Edmund. 1962. Ideas relativas a una fenomenología pura y una filosofía fenomenológica. México D.F: Fondo de Cultura Económica. Trad. de Gaos, José.

Husserl, Edmund. 1999. Investigaciones lógicas. Madrid: Alianza Editorial. Trad. de Morente, Manuel y Gaos, José.

Husserl, Edmund. 2008. La crisis de las ciencias europeas y la fenomenología trascendental. Buenos Aires: Prometeo Libros. Traducción de Iribarne, Julia.

Leyte, Arturo. 1996. “A propósito de Holzwege”. Isegoría, 13: 184-193.

Muñoz, Rubén. 2006. Tratamiento ontológico del silencio en Heidegger. Sevilla: Fénix editora.

Parente, Diego. 2006. "Tecnología y Gelassenheit: Heidegger y la apertura de futuros artificiales alternativos. Argumentos de Razón y Técnica 9: 37-61.

Pulido, Jorge. 2013. "Ser-aparecer-comprender". Praxis filosófica, 36: 225-249.

Pulido, Jorge. 2015. “<<La ontología solo es posible como fenomenología >>. En torno a la fenomenología de Martin Heidegger”. Franciscanum, 163: 87123.

Rivera, Jorge. 1998. "El silencio originario en el pensar de Heidegger". Estudios públicos, 69: 369-383. 
Shürmann, Reiner. 1973. "Heidegger and Meister Eckhart on Releasement". Research in Phenomenology, 3: 95-119. 